\title{
Insurance Status Is Related to Receipt of Therapy and Survival in Patients with Early-Stage Pancreatic Exocrine Carcinoma
}

\author{
Emily Boevers, ${ }^{1}$ Bradley D. McDowell, ${ }^{2}$ Sarah L. Mott, ${ }^{3}$ \\ Anna M. Button, ${ }^{3}$ and Charles F. Lynch ${ }^{4}$ \\ ${ }^{1}$ Carver College of Medicine, University of Iowa, Iowa City, IA 52242, USA \\ ${ }^{2}$ Holden Comprehensive Cancer Center Population Research Core, University of Iowa, Iowa City, IA 52242, USA \\ ${ }^{3}$ Holden Comprehensive Cancer Center Biostatistics Core, University of Iowa, Iowa City, IA 52242, USA \\ ${ }^{4}$ College of Public Health, Department of Epidemiology, University of Iowa, Iowa City, IA 52242, USA
}

Correspondence should be addressed to Emily Boevers; emily-boevers@uiowa.edu

Received 1 December 2016; Revised 8 March 2017; Accepted 29 March 2017; Published 10 April 2017

Academic Editor: Lance A. Liotta

Copyright (C) 2017 Emily Boevers et al. This is an open access article distributed under the Creative Commons Attribution License, which permits unrestricted use, distribution, and reproduction in any medium, provided the original work is properly cited.

\begin{abstract}
Objectives. The study objective was to determine how insurance status relates to treatment receipt and overall survival for patients with early-stage pancreatic exocrine carcinoma. Methods. SEER data were evaluated for 17,234 patients diagnosed with Stage I/II pancreatic exocrine carcinoma. Multivariate regression models controlled for personal characteristics to determine whether insurance status was independently associated with overall survival and receipt of radiation/surgery. Results. Odds of receiving radiation were 1.50 and 1.75 times higher for insured patients compared to Medicaid and uninsured patients, respectively $(p<0.01)$. Insured patients had 1.68 and 1.57 times increased odds of receiving surgery compared to Medicaid and uninsured patients $(p<0.01)$. Risk of death was 1.33 times greater $(p<0.01)$ in Medicaid patients compared to insured patients; when further adjusted for treatment, the risk of death was attenuated but remained significant (HR $=1.16, p<0.01)$. Risk of death was 1.16 times higher for uninsured patients compared to insured patients $(p=0.02)$; when further adjusted for treatment, the risk of death was no longer significant ( $\mathrm{HR}=1.01, p=0.83)$. Conclusions. Uninsured and Medicaid-insured patients experience lower treatment rates compared to patients who have other insurances. The increased likelihood of treatment appears to explain the insured group's survival advantage.
\end{abstract}

\section{Introduction}

Average survival time for pancreatic exocrine carcinoma patients is among the poorest of all cancer types. Recent years have seen little improvement [1], with pancreatic cancer expected to become the second leading cause of cancerrelated death by 2030 [2]. Although there are effective treatments for early-stage disease [3], many patients do not receive them. A minority of patients with early-stage disease undergo pancreatic resection, for example, even though it is a first-line therapy and is associated with improved survival [48]. Determining the reason for this could inform strategies to increase treatment rates and improve survival.

Disparities in insurance coverage may play an important role. It is known that patients with cancer at common sites (e.g., breast, colon, and lung) who lack insurance coverage have worse survival and inadequate treatment when compared to patients with insurance coverage [9]. Shapiro et al. used data from the Surveillance, Epidemiology, and End Results (SEER) program to establish that the same relationship exists for surgical treatment of pancreatic adenocarcinoma [10]. A SEER Patterns of Care study similarly showed that pancreatic cancer patients were more likely to receive cancer-directed surgery if they were insured [11]. Furthermore, two studies covering separate states have shown that patients with Medicaid or government-subsidized insurance have lower rates of cancer-directed surgery compared to those with private insurance $[12,13]$.

Disparities for those specifically covered by Medicaid have not been examined using the SEER dataset, the nation's 
only multistate, population-based cancer resource that contains comprehensive clinical and survival data [14]. The goal of our study was to use this resource to examine whether types of insurance are associated with treatment and survival disparities for pancreatic cancer patients.

\section{Materials and Methods}

Data from the 2016 release of the Surveillance, Epidemiology, and End Results (SEER) research dataset were used in this analysis [15]. The study cohort comprised 17,234 patients diagnosed with AJCC 6th Edition Stage IA/IB/IIA/IIB pancreatic exocrine carcinomas (ICD-O-3 site codes: C25.0, C25.1, and C25.2; histology codes: 8000, 8010, 8020, 8021, $8022,8140,8141,8144,8145,8230,8255,8440,8470,8480$, $8481,8490,8500,8521$, and 8560) between the years 2007 and 2013. Cases must have had known age/resection status and not be diagnosed at autopsy or by death certificate. Cases with unknown insurance status were also excluded, leaving the following SEER Insurance Recode categories for analysis: insured (which SEER created by combining private insurance, Medicare, other government insurance plans, and unspecified insurance), uninsured, and Medicaid coverage.

Logistic regression models were utilized to assess the association between insurance status and receipt of radiation or surgery (considered separately) while adjusting for age, sex, race, year of diagnosis, and stage. Estimated effects of predictors are reported as odds ratios (OR) along with 95\% confidence intervals. Cox regression was used to determine whether insurance status was a prognostic indicator of overall survival (OS) while adjusting for age, sex, race, year of diagnosis, and stage. Time was calculated from date of diagnosis to all-cause death. Patients still alive at the end of 2013 were censored. Estimates of predictors are reported as hazard ratios (HR) along with 95\% confidence intervals. All tests were two-sided and performed at the 5\% significance level using SAS v9.4 (SAS Institute, Cary, NC).

\section{Results}

3.1. Insurance Status and Patient Characteristics. Patients' characteristics and disease variables are shown in Table 1 . The majority of patients had Stage II disease $(77.9 \%, n=13,411)$. Most of the patients were white $(81.8 \%, n=14,090)$, and $51.1 \%$ of the patients were female $(n=8,799)$. Radiation therapy was provided to $30.4 \%$ of patients $(n=5,237$ ), and $47.6 \%$ of patients underwent surgery at the primary cancer site $(n=8,210)$. Most patients were insured by private insurance, Medicare, or other government insurance plans $(87.0 \%, n=$ $14,997)$; others were covered by Medicaid $(10.8 \% ; n=1,860)$ or were uninsured $(2.2 \% ; n=377)$.

To ensure that Medicare eligibility status did not affect the results, the interaction between age ( $<65$ and $65+$ years) and insurance status was examined in all models described in Table 1. This interaction between insurance status and age was found to be practically and statistically nonsignificant in all models. Thus, results from the more parsimonious, noninteraction models are reported Table 1.
3.2. Type of Insurance and Odds of Therapy. Table 2 illustrates the relationship between type of insurance and the odds of receiving radiation therapy or surgery after adjusting for age at diagnosis, year of diagnosis, race, sex, and stage. Insured patients had 1.50 and 1.75 times increased odds of receiving radiation compared to Medicaid and uninsured patients, respectively (both $p<0.01$ ). Similarly, insured patients were more likely to undergo surgery compared to Medicaid and uninsured patients (odds ratios of 1.68 and 1.57, resp.; both $p<0.01)$. There were no significant differences between the uninsured patients and those with Medicaid coverage for either radiation or surgical treatment.

3.3. Multivariate Survival Analysis. Table 3 shows survival as a function of insurance status adjusted for age at diagnosis, year of diagnosis, race, sex, and stage. The risk of death was 1.33 times greater $(p<0.01)$ in Medicaid patients compared to insured patients. Uninsured patients were at a higher risk of death compared to insured patients $(\mathrm{HR}=1.16, p=0.02)$. A borderline significant $(p=0.05)$ finding was that Medicaid patients had poorer overall survival compared to uninsured patients $(\mathrm{HR}=1.14, p=0.05)$.

Table 3 also shows the results of a multivariate analysis relating survival to insurance status when further adjusted for receipt of radiation and/or surgery. The result was an attenuation of some of the HRs shown in Table 3 with risk of death reduced to 1.16 times greater $(p<0.01)$ in Medicaid patients compared to insured patients, with no indication of a survival benefit for uninsured versus insured patients ( $\mathrm{HR}=1.01, p=0.83)$. Once again, a borderline significant $(p=0.05)$ finding was that Medicaid patients had poorer overall survival compared to uninsured patients $(\mathrm{HR}=1.14$, $p=0.05)$.

\section{Discussion}

Pancreatic exocrine carcinomas are aggressive and deadly, but proven treatments exist for early-stage disease. Disparities in delivery of these curative or life-prolonging therapies present a major obstacle to improving patient survival [16]. We observed that uninsured and Medicaid-insured pancreatic exocrine carcinoma patients experience lower odds of receiving both radiation therapy and surgical resection compared to patients who have other insurances. We also observed no difference for receipt of resection or radiation between noninsured and Medicaid-insured patients. These findings have implications for the recent efforts to expand Medicaid programs with the aim to increase access to care [17], even though it is not necessarily true that expanding these programs as they currently exist will have the desired effect.

We also observed a relationship between insurance status and overall survival for pancreatic exocrine carcinoma. This relationship has been established for other types of cancers [ 9 , 18-22]. Our analysis further revealed that both the Medicaid and uninsured populations had a significantly higher risk of death than the insured population after controlling for other individual and disease factors. 


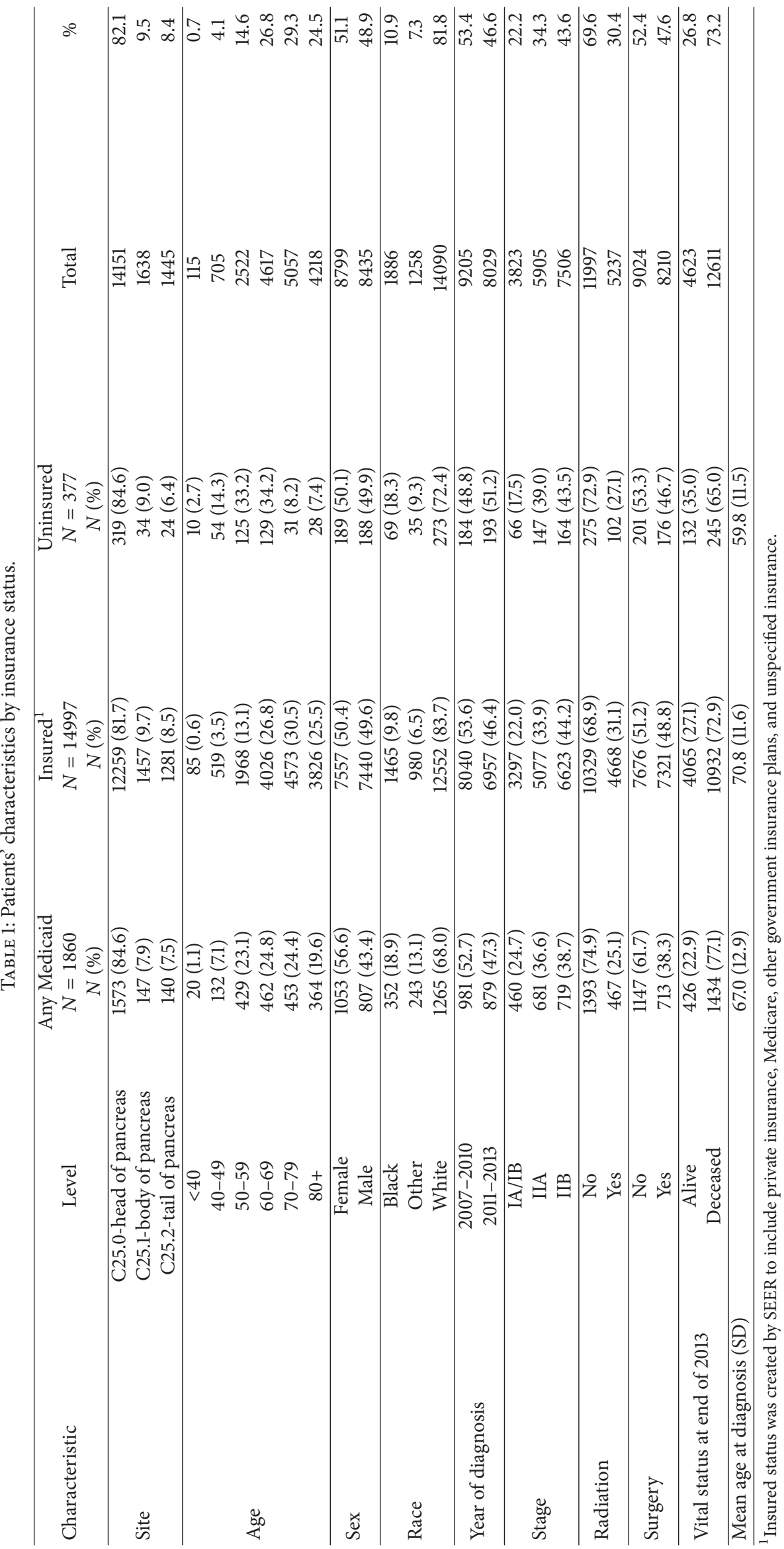


TABLE 2: Adjusted odds ratios for receiving radiation or surgery by insurance status.

\begin{tabular}{|c|c|c|c|c|c|c|}
\hline Outcome & Comparison & & OR & \multicolumn{2}{|c|}{$95 \% \mathrm{CI}$} & $P$ value \\
\hline \multirow{3}{*}{ Odds of receiving radiation ${ }^{1}$} & Insured & Versus any Medicaid & 1.50 & 1.34 & 1.68 & $<.01$ \\
\hline & Uninsured & Versus any Medicaid & 0.85 & 0.66 & 1.10 & 0.23 \\
\hline & Insured & Versus uninsured & 1.75 & 1.39 & 2.22 & $<.01$ \\
\hline \multirow{3}{*}{ Odds of undergoing surgery ${ }^{1}$} & Insured & Versus any Medicaid & 1.68 & 1.50 & 1.88 & $<.01$ \\
\hline & Uninsured & Versus any Medicaid & 1.07 & 0.84 & 1.37 & 0.58 \\
\hline & Insured & Versus uninsured & 1.57 & 1.25 & 1.97 & $<.01$ \\
\hline
\end{tabular}

${ }^{1}$ Adjusted for age at diagnosis, sex, year of diagnosis, race, and stage.

TABLE 3: Adjusted hazard ratios for all-cause survival by insurance status.

\begin{tabular}{|c|c|c|c|c|c|c|c|c|c|c|}
\hline \multirow[t]{3}{*}{ Outcome } & \multirow{2}{*}{\multicolumn{2}{|c|}{ Comparison }} & \multicolumn{4}{|c|}{ Controlled for personal characteristics } & \multicolumn{4}{|c|}{$\begin{array}{c}\text { Controlled for personal characteristics and } \\
\text { treatment received }\end{array}$} \\
\hline & & & \multirow{2}{*}{$\begin{array}{c}\mathrm{HR}^{1} \\
1.33\end{array}$} & \multicolumn{2}{|c|}{$95 \% \mathrm{CI}$} & \multirow{2}{*}{$\frac{P \text { value }}{<.01}$} & \multirow{2}{*}{$\begin{array}{c}\mathrm{HR}^{2} \\
1.16\end{array}$} & \multicolumn{2}{|c|}{$95 \% \mathrm{CI}$} & \multirow{2}{*}{$\frac{P \text { value }}{<.01}$} \\
\hline & Any Medicaid & Versus insured & & 1.25 & 1.40 & & & 1.10 & 1.23 & \\
\hline \multirow[t]{2}{*}{$\begin{array}{l}\text { Overall } \\
\text { survival }\end{array}$} & Any Medicaid & $\begin{array}{l}\text { Versus } \\
\text { uninsured }\end{array}$ & 1.14 & 1.00 & 1.31 & 0.05 & 1.14 & 1.00 & 1.31 & 0.05 \\
\hline & Uninsured & Versus insured & 1.16 & 1.02 & 1.32 & 0.02 & 1.01 & 0.89 & 1.15 & 0.83 \\
\hline
\end{tabular}

${ }^{1}$ Adjusted for age at diagnosis, sex, year of diagnosis, race, and stage.

${ }^{2}$ Adjusted for age at diagnosis, sex, year of diagnosis, race, stage, radiation, and/or surgery.

It is especially interesting that the survival difference between the insured and the uninsured populations is absent when the analysis is controlled for receipt of therapy (HR $=1.01$ ). This suggests that improved survival for insured patients may exist because they are more likely to receive therapy. However, something different is observed for the Medicaid-insured population: these patients have poorer survival even after controlling for receipt of resection and radiation. This suggests that worse outcomes for this group are due to more than just lack of treatment. One explanation for poorer survival for Medicaid patients is that they may have poorer health status overall. Medicaid patients may also lack the provider options that privately insured patients have access to, and this could delay care or compromise expertise. Patients with Medicaid insurance or without insurance might experience less treatment or less aggressive care in other ways, which diminishes their survival potential, including less adherence to treatment guidelines [23, 24].

Unfortunately, the SEER research dataset does not include comorbidity data and other variables that may allow us to explore these possibilities, and this represents a limitation of the study. Another limitation of the SEER data is the lack of drug-specific chemotherapy data. Also, the relatively small sample size for the uninsured group could have affected the power of some of the analyses. An additional limitation is that the Insurance Recode variable provided in the SEER research dataset combines several insurance categories (i.e., private insurance, Medicare, other government insurance plans, and unspecified insurance), and it is possible that these subtypes have different associations with treatment and survival. A strength of the study is that it employs a very large cohort of patients from high-quality SEER registries that cover approximately $28 \%$ of the population of the United States [14].

Addressing insurance disparities may improve aggregate survival and individual patient outcomes using proven therapies. Specifically, increasing rates of pancreatectomy will likely increase the average survival of pancreatic exocrine carcinoma patients $[4,21]$. Greater adherence to accepted guidelines offers patients and providers the opportunities for improved outcomes [24]. Increased education among providers about the indications for treatment and improvements in survival is needed. Additionally, improved safeguards should be implemented to minimize the influence of nonclinical factors, including insurance status, on treatment planning decisions.

\section{Disclosure}

This paper was presented in part at the Academic Surgical Congress, February 2015, Las Vegas, NV.

\section{Conflicts of Interest}

The authors declare that they have no conflicts of interest.

\section{Acknowledgments}

This project was supported in part by the Holden Comprehensive Cancer Center Population Research Core and Biostatistics Core (P30 CA086862). The authors thank James J. Mezhir, M.D. (1973-2016), for his valued contribution to this research. 


\section{References}

[1] C. E. Rutter, H. S. Park, C. D. Corso et al., "Addition of radiotherapy to adjuvant chemotherapy is associated with improved overall survival in resected pancreatic adenocarcinoma: an analysis of the National Cancer Data Base," Cancer, vol. 121, no. 23, pp. 4141-4149, 2015.

[2] L. Rahib, B. D. Smith, R. Aizenberg, A. B. Rosenzweig, J. M. Fleshman, and L. M. Matrisian, "Projecting cancer incidence and deaths to 2030: the unexpected burden of thyroid, liver, and pancreas cancers in the united states," Cancer Research, vol. 74, no. 11, pp. 2913-2921, 2014.

[3] M. Ducreux, A. S. Cuhna, C. Caramella et al., "Cancer of the pancreas: ESMO Clinical Practice Guidelines for diagnosis, treatment and follow-up," Annals of Oncology, vol. 26, supplement 5, pp. v56-v68, 2015.

[4] B. D. McDowell, C. G. Chapman, B. J. Smith, A. M. Button, E. A. Chrischilles, and J. J. Mezhir, "Pancreatectomy predicts improved survival for pancreatic adenocarcinoma: results of an instrumental variable analysis," Annals of Surgery, vol. 261, pp. 740-745, 2015.

[5] Y. Shaib, J. Davila, C. Naumann, and H. El-Serag, "The impact of curative intent surgery on the survival of pancreatic cancer patients: a U.S. population-based study," American Journal of Gastroenterology, vol. 102, no. 7, pp. 1377-1382, 2007.

[6] R. L. Siegel, K. D. Miller, and A. Jemal, "Cancer statistics, 2015," CA Cancer Journal for Clinicians, vol. 65, no. 1, pp. 5-29, 2015.

[7] J. M. Winter, M. F. Brennan, L. H. Tang et al., "Survival after resection of pancreatic adenocarcinoma: results from a single institution over three decades," Annals of Surgical Oncology, vol. 19, no. 1, pp. 169-175, 2012.

[8] K. Y. Bilimoria, D. J. Bentrem, C. Y. Ko, A. K. Stewart, D. P. Winchester, and M. S. Talamonti, "National failure to operate on early stage pancreatic cancer," Annals of Surgery, vol. 246, no. 2, pp. 173-180, 2007.

[9] M. T. Halpern, E. M. Ward, A. L. Pavluck, N. M. Schrag, J. Bian, and A. Y. Chen, "Association of insurance status and ethnicity with cancer stage at diagnosis for 12 cancer sites: a retrospective analysis," The Lancet Oncology, vol. 9, no. 3, pp. 222-231, 2008.

[10] M. Shapiro, Q. Chen, Q. Huang et al., "Associations of socioeconomic variables with resection, stage, and survival in patients with early-stage pancreatic cancer," JAMA Surgery, vol. 151, no. 4, pp. 338-345, 2016.

[11] V. L. Shavers, L. C. Harlan, M. Jackson, and J. Robinson, "Racial/ethnic patterns of care for pancreatic cancer," Journal of Palliative Medicine, vol. 12, no. 7, pp. 623-630, 2009.

[12] A. Abraham, W. B. Al-Refaie, H. M. Parsons, V. Dudeja, S. M. Vickers, and E. B. Habermann, "Disparities in pancreas cancer care," Annals of Surgical Oncology, vol. 20, no. 6, pp. 2078-2087, 2013.

[13] A. P. Loehrer, D. C. Chang, M. M. Hutter et al., "Health insurance expansion and treatment of pancreatic cancer: does increased access lead to improved care?" Journal of the American College of Surgeons, vol. 221, no. 6, pp. 1015-1022, 2015.

[14] Surveillance Epidemiology and End Results Program (SEER) Program, Overview of the SEER Program, National Cancer Institute, 2016, https://seer.cancer.gov/about/overview.html.

[15] Surveillance Epidemiology and End Results Program (SEER) Program, "SEER*Stat Database: Incidence-SEER 9 Regs Research Data, Nov 2015 Sub (1973-2013), Katrina/Rita Population Adjustment, Linked to County Attributes-Total U.S., 1969-2014 Counties, National Cancer Institute, DCCPS,
Surveillance Research Program, Surveillance Systems Branch," April 2016.

[16] S. Amin, A. L. Lucas, and H. Frucht, "Evidence for treatment and survival disparities by age in pancreatic adenocarcinoma: a population-based analysis," Pancreas, vol. 42, no. 2, pp. 249-253, 2013.

[17] V. Wachino, S. Artiga, and R. Rudowitz, How is the ACA Impacting Medicaid Enrollment, The Kaiser Commission on Medicaid and the Uninsured, Kaiser Family Foundation, Menio Park, Calif, USA, 2014.

[18] C. J. Bradley, C. W. Given, and C. Roberts, "Race, socioeconomic status, and breast cancer treatment and survival," Journal of the National Cancer Institute, vol. 94, no. 7, pp. 490-496, 2002.

[19] O. Dejardin, A. M. Bouvier, J. Faivre, S. Boutreux, G. De Pouvourville, and G. Launoy, "Access to care, socioeconomic deprivation and colon cancer survival," Alimentary Pharmacology and Therapeutics, vol. 27, no. 10, pp. 940-949, 2008.

[20] K. M. Gorey, "Breast cancer survival in Canada and the USA: meta-analytic evidence of a Canadian advantage in low-income areas," International Journal of Epidemiology, vol. 38, no. 6, pp. 1543-1551, 2009.

[21] K. McDavid, T. C. Tucker, A. Sloggett, and M. P. Coleman, "Cancer survival in Kentucky and health insurance coverage," Archives of Internal Medicine, vol. 163, no. 18, pp. 2135-2144, 2003.

[22] R. Shi, G. Mills, J. McLarty, G. Burton, Z. Shi, and J. Glass, "Commercial insurance triples chances of breast cancer survival in a public hospital," Breast Journal, vol. 19, no. 6, pp. 664-667, 2013.

[23] E. Ward, M. Halpern, N. Schrag et al., "Association of insurance with cancer care utilization and outcomes," CA: A Cancer Journal for Clinicians, vol. 58, no. 1, pp. 9-31, 2008.

[24] L. C. Harlan, A. L. Greene, L. X. Clegg, M. Mooney, J. L. Stevens, and M. L. Brown, "Insurance status and the use of guideline therapy in the treatment of selected cancers," Journal of Clinical Oncology, vol. 23, no. 36, pp. 9079-9088, 2005. 


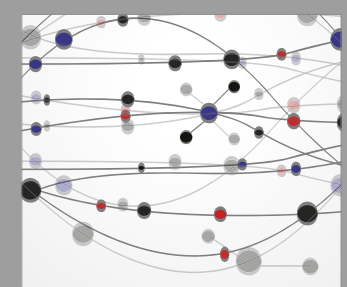

The Scientific World Journal
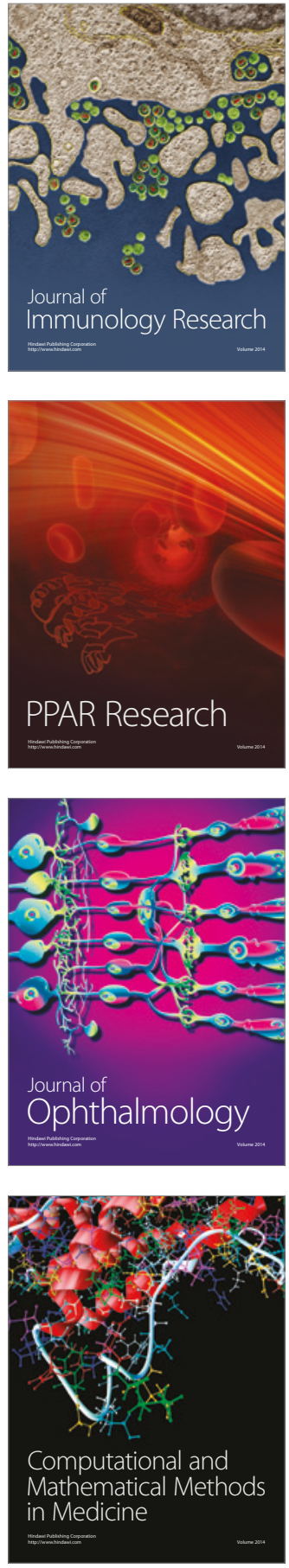

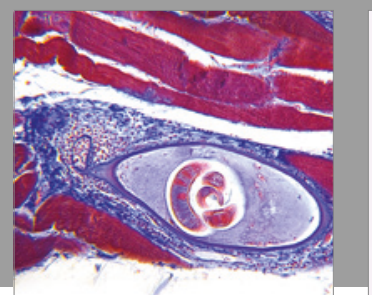

Gastroenterology Research and Practice
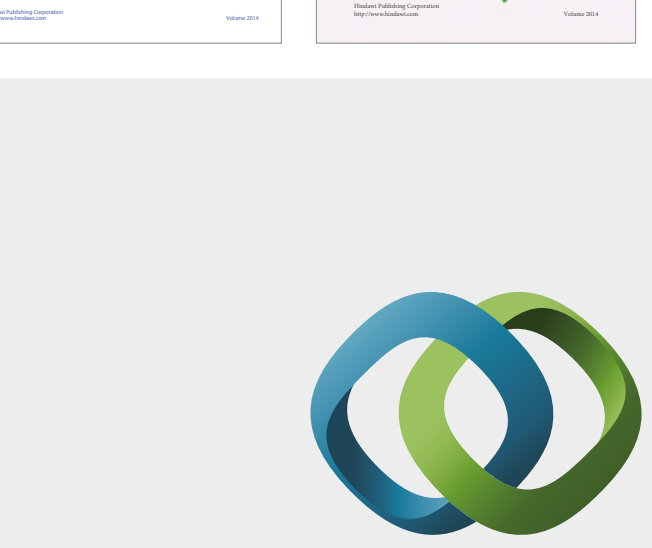

\section{Hindawi}

Submit your manuscripts at

https://www.hindawi.com
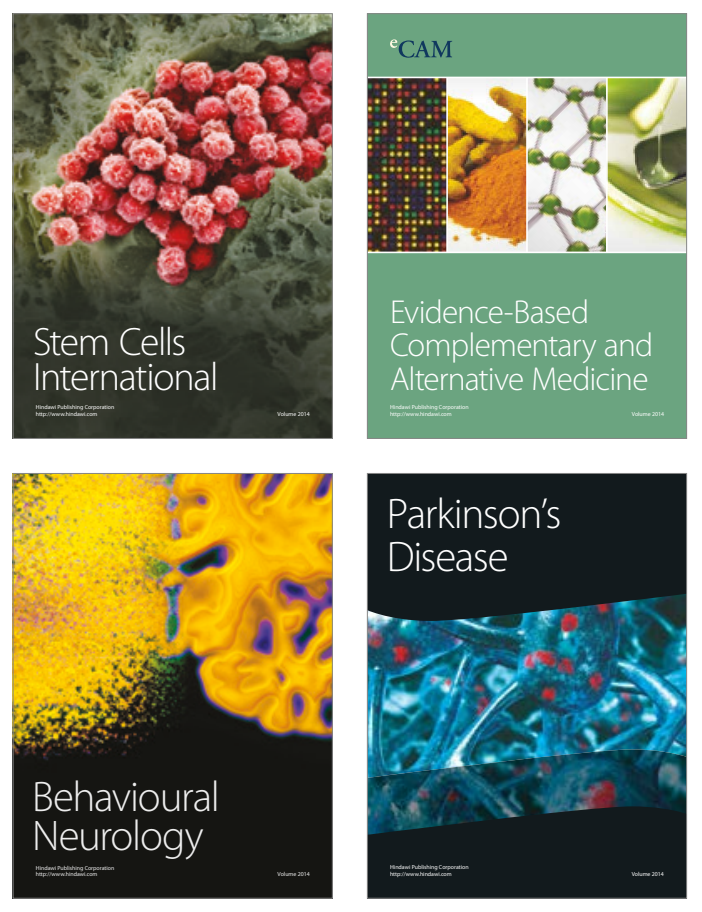
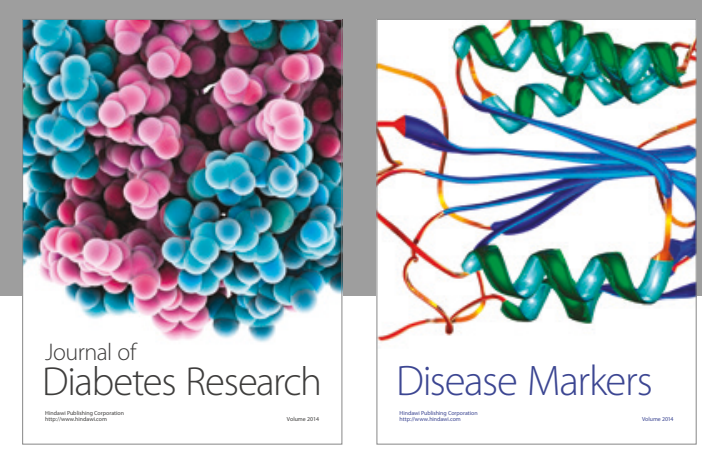

Disease Markers
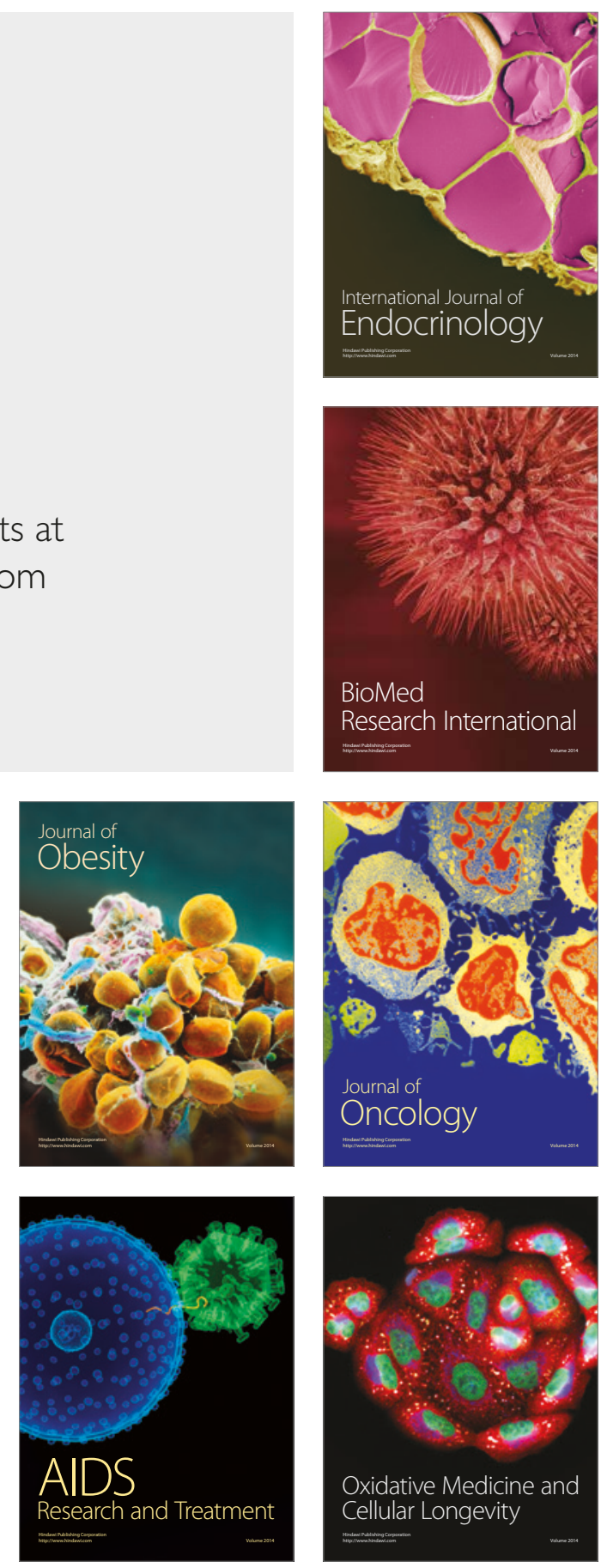